\title{
Cooperative Information Fusion in a Network Robot System
}

\author{
Kevin LeBlanc and Alessandro Saffiotti \\ AASS Mobile Robotics Lab, Örebro University, Örebro, Sweden \\ http://aass.oru.se/Research/Robots/ \\ $\{$ klc,asaffio\}@aass.oru.se
}

\begin{abstract}
The vision of network robot systems involves robots embedded in smart environments, with which they can collaborate and communicate. In such systems, robots have access to many different sources and types of information, which need to be coordinated and combined effectively. In this work, we propose a framework which addresses the problem of combining heterogeneous information in a network robot system. The approach is inspired by work on conceptual spaces and perceptual anchoring, and is implemented using tools from fuzzy logic.
\end{abstract}

\section{INTRODUCTION}

When dealing with network robot systems, as opposed to standalone robotic systems, one of the main new aspects is the unprecedented richness of information available. In these systems, agents do not need to rely solely on their own perception to obtain information about objects: they can also receive information from the objects themselves, or from other agents with knowledge about objects in the environment. Moreover, various types of information are often available; for example, an agent might have access to multiple sources of perceptual and non-perceptual information. While this richness of information opens a new landscape of opportunities, it also adds a number of fundamental challenges - namely, those of communicating, coordinating and fusing these items of information.

A number of approaches to highly distributed robotic systems exist, under different names, including: network robot systems [1], intelligent spaces [2], sensor-actuator networks [3], ubiquitous robotics [4], and PEIS-Ecologies [5]. Most of the approaches have much in common, and all of them need to address the challenges mentioned above. In the rest of this paper we will refer to these systems as PEIS-Ecologies, since this work was carried out from within this context.

We propose a framework for fusing different types of information obtained from various sources. If done correctly, information fusion can yield improved knowledge about the state of the world. Redundant information can be used to reduce uncertainty and imprecision, while complementary information can be used to resolve ambiguities and incompleteness. The work is inspired by work on perceptual anchoring [6],

This work is supported in part by CUGS (the Swedish computer graduate school), and in part by ETRI (Electronics and Telecommunications Research Institute, Korea) through the project "Embedded Component Technology and Standardization for URC (2004-2008)".
[7], [8] and conceptual spaces [9], and as such it is well suited for dealing with both perceptual and non-perceptual (often symbolic) information. The framework uses geometric spaces, called anchoring spaces, to represent, compare and combine information. The instance of the framework described here uses tools from fuzzy logic to represent, match, and fuse various items of information. The main contribution of the framework is that it allows the many different types of information which are present in robotic systems to be efficiently represented, matched and fused using a single conceptual space representation. More details on the framework, as well as a discussion on related work, can be found in [10].

\section{INFORMATION FUSION IN A PEIS-ECOLOGY}

In a PEIS-Ecology [5], all agents which are members of the ecology are abstracted by the uniform notion of a PEIS (Physically Embedded Intelligent System), which is any device incorporating some computational and communication resources, and possibly able to interact with the environment via sensors and/or actuators. A PEIS can be as simple as a toaster or as complex as a humanoid robot. Our general framework for fusion within a PEIS-Ecology can thus be summarized as follows. Consider a set $P=\left\{p_{1}, \ldots, p_{n}\right\}$ of PEIS. For each PEIS $p_{i}$ in $P$, we define:

- An anchoring space $S_{i}$. This is a multi-dimensional metric space. Dimensions correspond to qualities of interest in the application domain; i.e., groups of dimensions are related to an object's interesting properties.

- A set $X_{i}=\left\{x_{i}^{1}, \ldots, x_{i}^{k}\right\}$ of information sources for each PEIS $p_{i}$. For a typical PEIS, a local symbol system (e.g. a knowledge base, or a task planner), and a number of sensing modalities could all act as information sources.

- For each source $x_{i}^{j}$, we define a grounding function $g_{i}^{j}$ which maps information from the given source into the anchoring space $S_{i}$. For example a source containing symbolic information might have a grounding function which maps predicate symbols to regions in $S_{i}$. Alternatively, a grounding function might map percepts from a perceptual system to areas or points in $S_{i}$.

- A shared anchoring space $S$, which is the space into which shared information, obtained from various PEIS, is mapped. This shared space provides a common reference frame, which is needed in order for communicated 


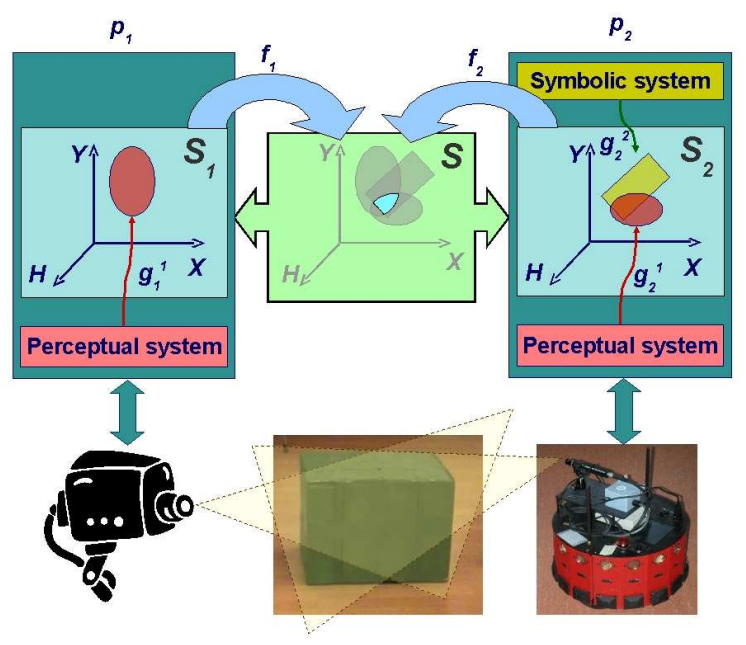

Fig. 1. A simple PEIS-Ecology containing two PEIS.

information to be compared, matched, and fused.

- For each PEIS $p_{i}$, a function $f_{i}: S_{i} \rightarrow S$ which maps information from $S_{i}$ to $S$. The functions $f_{i}$ will sometimes involve cylindrical extensions and non-linear transformations, since the dimensionality and coordinate systems of $S$ and $S_{i}$ might differ.

\section{A. A simple example}

Imagine a simple PEIS-Ecology like the one illustrated in Figure 1, which consists of two PEIS: an autonomous robot called Pippi, endowed with a symbolic system (e.g., a task planner) and a perceptual system (e.g., a vision system); and a camera which also has a perceptual system, but no symbolic system. Both PEIS are observing the same object, a green box.

For simplicity, we assume that both PEIS have individual anchoring spaces which have the same dimensions (e.g. X, $\mathrm{Y}$, and Hue) as the shared anchoring space (which is shown above the box in the figure). This means that each PEIS can directly map information obtained from their information sources into the shared space. If a PEIS makes observations in local robot coordinates, a coordinate transformation to global coordinates might be needed in order to map information into the coordinate system used in the shared anchoring space. Note that in practice, position and color information would be maintained separately, to reduce computational complexity.

Before the information in the shared space can be fused, one must determine which items of information refer to the same objects. This data-association is non-trivial, and is part of the anchoring problem [6]. For this work, it suffices to note that comparing and matching information can be done conveniently inside conceptual spaces.

Once it is known which items of information refer to a given object (in this case, the green box), the information in the shared space can be fused, resulting in an improved estimate of the box's position and hue. The fusion process will be discussed in more detail in the next section.

\section{A FuZZY LOGIC IMPLEMENTATION}

We have implemented the framework described previously using techniques from fuzzy logic [11]. Information in the individual and shared anchor spaces is represented by $n$ dimensional fuzzy sets. Simply put, a fuzzy set $A$ over the $n$-dimensional space $S$ is characterized by a membership function

$$
\mu_{A}: S \rightarrow[0,1]
$$

which gives the degree of membership $\mu_{A}(s)$ of each point $s$ in $S$ to the fuzzy set $A$. This means that the grounding function $g_{i}^{j}$ maps information from the information source $x_{i}^{j}$ to a fuzzy set over the individual anchor space $S_{i}$.

Each fuzzy set $A$ is implemented as an $n$-dimensional array of floats, where the value in each element $s$ corresponds to the value of $\mu_{A}(s)$. For example, color information can be represented using a 3D space, with dimensions for hue, saturation, and lightness, while $2 \mathrm{D}$ position information can be represented using a grid of $(x, y)$ values. If necessary, some dimensions can be approximated to reduce complexity.

In the implementation used in this work, the dimensionality and units of the shared space $S$ are the same as those used in the individual spaces $S_{i}$. This implies that for each PEIS $p_{i}$, the mapping function $f_{i}$ which maps information from the individual anchor space $S_{i}$ to the shared anchor space $S$ is the identity function. This choice does not limit the generality of the framework; it merely simplifies the implementation, by affecting how and when certain computations are made.

The shared space could be implemented in a centralized or distributed way. In this work, the shared space is distributed: each PEIS has a local copy of $S$, in which it performs matching and fusion operations.

Information fusion is implemented using fuzzy intersection. If $\mu_{1}$ and $\mu_{2}$ are two fuzzy sets on the same space $S$, representing two distinct items of information, then the result of fusing these two items is represented by the fuzzy set $\mu$ given, for any $s \in S$, by

$$
\mu(s)=\mu_{1}(s) \otimes \mu_{2}(s),
$$

where $\otimes$ is a triangular norm, or T-norm [11]. The most common T-norms used in fuzzy logic are the minimum and product operators; in the experiments reported below, we use the product. Figure 2 shows an example of two 1-dimensional fuzzy sets being fused using the product T-norm. Information matching is also implemented using the mechanisms of fuzzy logic. Various definitions for fusion and matching might be used, and several alternatives exist in the fuzzy logic literature.

\section{AN ILlustrative EXPERIMENT}

The purpose of this experiment is to demonstrate how information of different types (in this case symbolic and perceptual), and from different sources, can be fused using a common representation space. This yields two main benefits:

- disambiguation: when multiple hypotheses about the state of an object are possible, or when multiple percepts could be associated with a certain object, complementary 


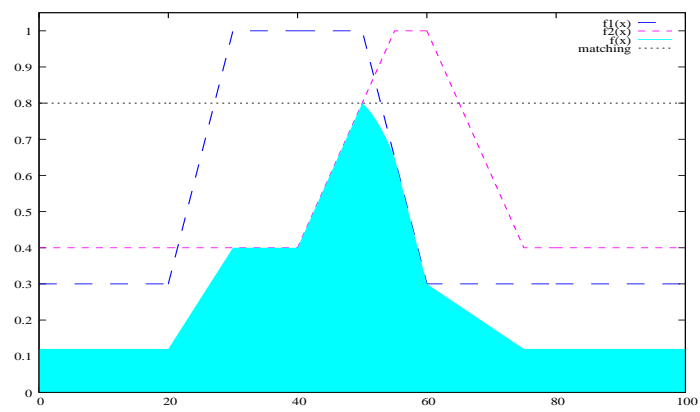

Fig. 2. The fuzzy set $f(x)$ is the result of fusing $f 1(x)$ and $f 2(x)$ using the product T-norm. One way to compute a matching level is to take the maximum point of $\mathrm{f}(\mathrm{x})$, which is approximately 0.8 .

information from another source can aid in isolating the correct hypothesis;

- improved estimates: when multiple sources of information are considered, redundancy in information can be used to reduce imprecision and inaccuracy.

The environment we use for developing and testing PEISEcologies in various configurations is a small apartment, which we call the PEIS-Home. The experiment described here is based on a coarse 2D model of the PEIS-Home, shown in Figure 3(a). This model includes two rooms (a kitchen and a bedroom), as well as two tables. One of the tables, Table01, is a PEIS, and possesses an RFID reader which can read the RFID tags of any object placed on its surface. There are two other PEIS used in this experiment. One is an overhead camera, placed in the kitchen, called Camera-01. The other is the robot Pippi, initially assumed to be in the bedroom.

In this discussion, the only dimensions of the anchoring spaces which are considered are $X$ and $Y$ (i.e. we deal only with 2D positions). Recall that the individual and shared anchoring spaces have the same dimensions and units here. In practice this means that all position information obtained in local coordinates (e.g., from Pippi's vision system) is converted to global coordinates before being put into the anchoring space. This is done using the grounding functions described earlier.

This experiment is carried out using artificially generated data, and demonstrates a simple scenario involving the above mentioned PEIS. Using artificial data in this case allows us to carefully examine new items of information one at a time. In [12], the fusion algorithm itself was examined using online data. The scenario unfolds as follows.

- Information 1 (Symbolic): Pippi is told to fetch a green cup, called Cup-22, from the kitchen. Figure 3(b) shows the fuzzy set used by Pippi to represent the information that the cup is in the kitchen (i.e. positions in the kitchen are possible, all other positions are impossible).

- Information 2 (Perceptual): Pippi asks the ecology for information about all cups. Camera-01 sees two green cups in the kitchen, which are the only cups in the ecology which match the information Pippi already has about Cup-22. The fuzzy set showing the positions of the cups perceived by Camera-01 is shown in Figure 3(c). The result of fusing this information with previous information is shown in Figure 3(d).

- Information 3 (Symbolic): Table-01 detects that Cup-22 is on its surface with its RFID reader (Figure 3(e)). The result of fusing this information with previous information is shown in Figure 3(f). Note that this has led to disambiguation, since only one of the cups observed by the camera has a position which is consistent with that of the table.

- Information 4 (Perceptual): Pippi now has enough information to approach $\mathrm{Cup}-22$, and can thus perceive the cup with an on-board camera (Figure 3(g)). The result of combining all of the available information is shown in Figure 3(h). Combining the information from both cameras, which have different viewpoints, improves the final estimate considerably, since range estimates from the individual cameras are quite uncertain.

\section{Conclusions}

We have described a framework for fusing the information provided by several heterogeneous sources of information in a multi-robot setting. We have shown in particular an instantiation of this framework within PEIS-Ecologies, a type of network robot system, as well as an implementation of it based on fuzzy logic. Though other techniques might be used to implement the framework, the fuzzy logic approach has a number of important advantages. The anchoring spaces used in our fusion framework provide a general way of representing and treating various types of information.

\section{REFERENCES}

[1] "Network Robot Forum," www.scat.or.jp/nrf/English/.

[2] J. Lee and H. Hashimoto, "Intelligent space - concept and contents," Advanced Robotics, vol. 16, no. 3, pp. 265-280, 2002.

[3] F. Dressler, "Self-organization in autonomous sensor/actuator networks," in Proc of the 19th IEEE Int Conf on Architecture of Computing Systems, 2006.

[4] J. Kim, Y. Kim, and K. Lee, "The third generation of robotics: Ubiquitous robot," in Proc of the 2nd Int Conf on Autonomous Robots and Agents, Palmerston North, New Zealand, 2004.

[5] A. Saffiotti and M. Broxvall, "PEIS ecologies: Ambient intelligence meets autonomous robotics," in Proc of the Int Conf on Smart Objects and Ambient Intelligence, Grenoble, France, 2005, pp. 275-280.

[6] S. Coradeschi and A. Saffiotti, "An introduction to the anchoring problem," Robotics and Autonomous Systems, vol. 43, no. 2-3, pp. 8596, 2003.

[7] A. Chella, S. Coradeschi, M. Frixione, and A. Saffiotti, "Perceptual anchoring via conceptual spaces," in Proc. of the AAAI-04 Workshop on Anchoring Symbols to Sensor Data, San Jose, CA, 2004.

[8] K. LeBlanc and A. Saffiotti, "Issues of perceptual anchoring in an ubiquitous robotic system," in Proc of the ICRA-07 Workshop on Omniscient Space, Rome, Italy, 2007.

[9] P. Gärdenfors, Conceptual Spaces: The Geometry of Thought. Cambridge, MA, USA: MIT Press, 2000.

[10] K. LeBlanc, "Cooperative perceptual anchoring using conceptual spaces," Ph.D. dissertation, Örebro University, Örebro, Sweden, 2008, in preparation.

[11] G. J. Klir and T. A. Folger, Fuzzy sets, uncertainty, and information. Prentice-Hall, 1988

[12] J.-P. Cánovas, K. LeBlanc, and A. Saffiotti, "Robust multi-robot object localization using fuzzy logic," in RoboCup 2004, D. Nardi, M. Riedmiller, and C. Sammut, Eds. Berlin, DE: Springer-Verlag, 2004, pp. 247-261. 


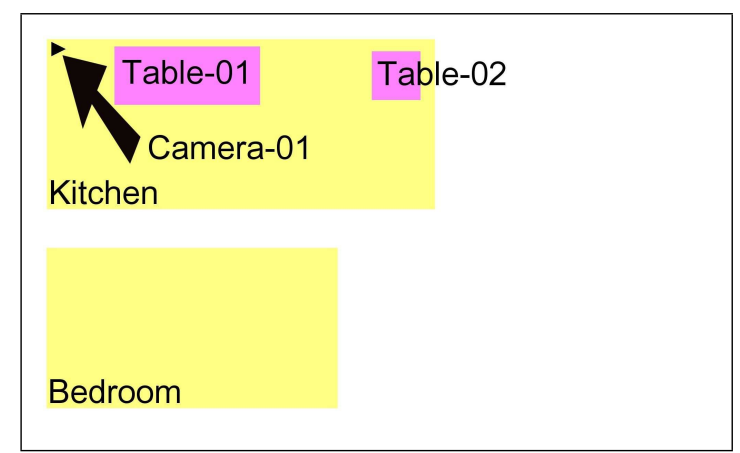

(a) A coarse model of the PEIS apartment.

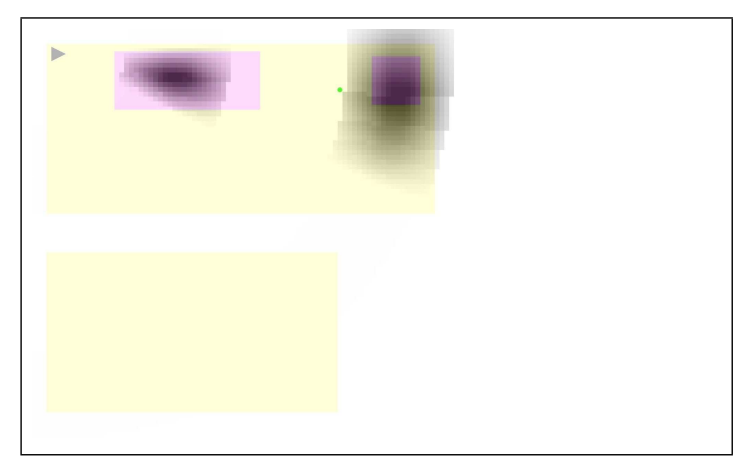

(c) Information 2: Camera-01 sees two green cups.

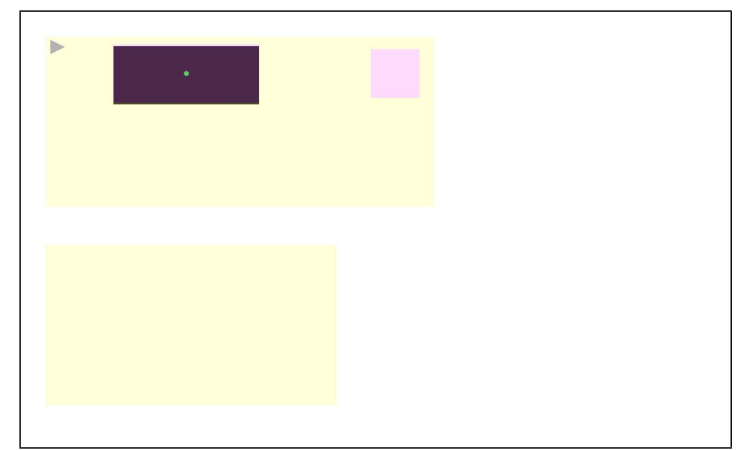

(e) Information 3: Table-01 detects Cup-22.

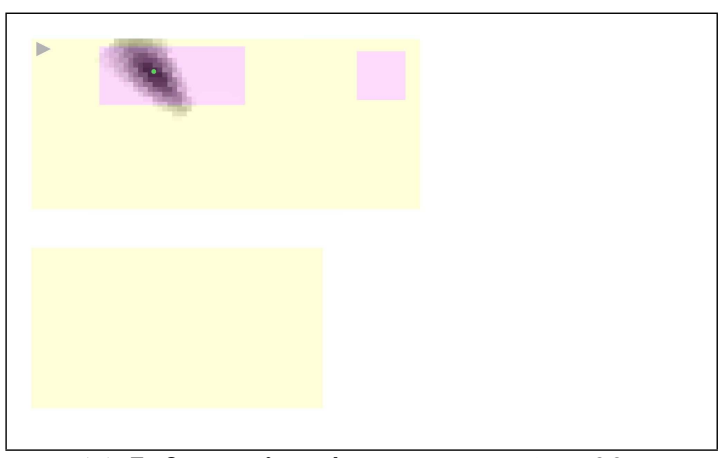

(g) Information 4: Pippi sees Cup-22.

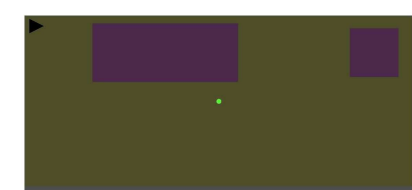

(b) Information 1: Cup-22 is in the kitchen.

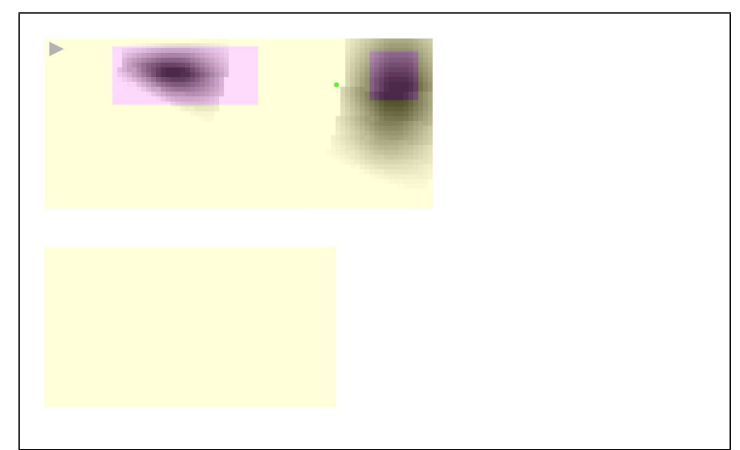

(d) Information 1,2: Combination of items 1 and 2.

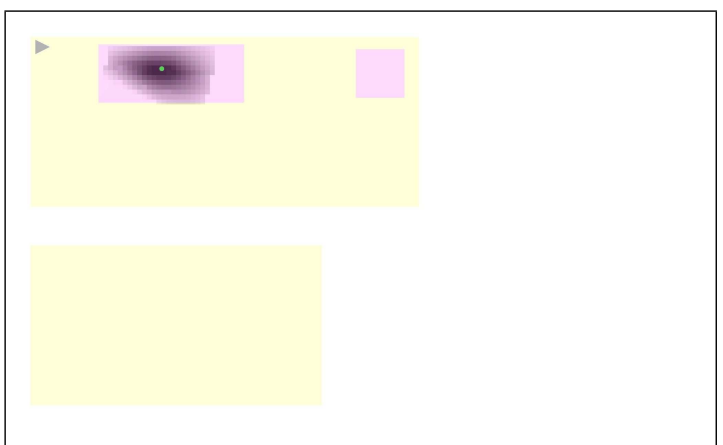

(f) Information 1,2,3: Combination of items 1, 2 and 3.

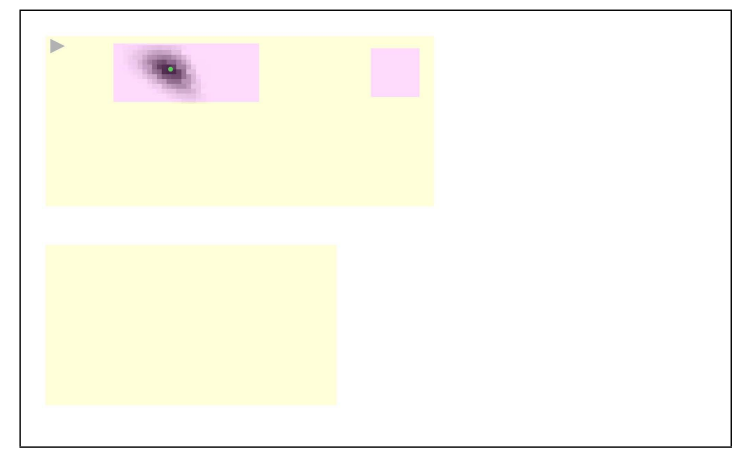

(h) Information 1,2,3,4: Final position estimate.

Fig. 3. Progression of estimates for the position of Cup-22, as new items of information arrive. The darker areas are more possible, the light areas are less possible. The dots indicate the center of gravity of the possible areas. 